\title{
Selective attention to global and local structure of objects: Alternative measures of nontarget processing
}

\author{
KEVIN A. BRIAND \\ State University of New York, Albany, New York
}

\begin{abstract}
When individuals are asked to report either the global or the local level of structure in a stimulus pattern located inside a relevant object, distractors located within an irrelevant object will interfere only if they are at the same level of structure as that of the target item (Briand, 1993; Paquet \& Merikle, 1988). The basis of this level-specific filtering is unclear, as is the true level of semantic analysis for the ignored items. In the present series of experiments, multiple measures of nontarget processing were used to assess concurrent interference, negative priming, and a category effect supposedly reflecting a more abstract level of semantic analysis. These different indicants were assessed in three experiments in which form, color, or a spatial precue was used to direct attention to the relevant stimulus pattern. Overall, cuing by form produced poorer spatial selectivity, whereas spatial precues and color led to better selectivity. However, the three measures of nontarget processing were not equally affected by these manipulations, with global information showing more evidence for semantic analysis than for local analysis regardless of the type of selection cue used. The results suggest that inhibition is not the basis of target selection when ignored items are local, but that it may be used when global items are ignored.
\end{abstract}

Paquet (1988; Paquet \& Merikle, 1988) reported a series of experiments on selective attention to either the global or the local level of structure of a relevant object. While performing this task, subjects were required to ignore possible target letters (either global or local) appearing in a simultaneously displayed object. This paradigm is an extension of a similar task developed by Kahneman and Henik (1981). In the newer paradigm, two compound letter patterns (Navon, 1977) were presented, one enclosed within a circular outline and another within a square one. The subjects were required to identify the target letter at a predesignated level of structure within a predesignated shape. The major result observed in this paradigm were that concurrent interference from letters contained within the irrelevant shape only occurred when they were at the same level of structure as that of the target (Briand, 1993; Briand \& Klein, 1993; Paquet, 1988; Paquet \& Merikle, 1988).

To determine the extent of processing of unattended information, it is common to manipulate semantic or identity information of the unattended items. If there is no effect

The experiments reported here were carried out in partial fulfillment of the PhD degree requirements at Dalhousie University. The research was supported by an NSERCC Graduate Scholarship and a Killam Fellowship to the author, as well as by an NSERCC Research Grant to R. M. Klein. Requests for reprints should be addressed to the author at the Department of Psychology, University at Albany, State University of New York, Albany, NY 12222.

-Accepted by previous editor, Charles W. Eriksen of their identity on target processing, it is assumed that unattended items are not identified. Duncan (1981) has criticized this logic by pointing out that even though selection may be efficient (i.e., ignored items have little effect on responses to targets), this does not imply that the unselected items have not been analyzed semantically. However, it is equally clear that the influence of unattended items is clear evidence that those items have been analyzed to at least the semantic level. A major problem is that most studies of selective attention have involved only one measure of semantic processing-that is, Stroop-type interference with the processing of the attended items as a function of the identity of the unattended items, or, in other words, whether the response indicated by this unattended item is compatible or incompatible with the response indicated by the attended item (see, e.g., Eriksen \& Eriksen, 1974; Kahneman \& Henik, 1981; Paquet \& Merikle, 1988). An obvious weakness of any study of selective attention that relies on one measure of the processing of unattended items is that the failure to find an effect of their identity does not mean that they have not been identified. It is entirely conceivable that some other measures might have revealed extensive semantic analysis (Allport, Tipper, \& Chmiel, 1985; Driver \& Tipper, 1989).

These points are clearly relevant in the context of the paradigm described initially. One of the striking findings observed in this paradigm (Briand \& Klein, 1993; Paquet $\&$ Merikle, 1988) is that when subjects select the relevant object on the basis of the shape of the outline surrounding the letter pattern, unattended letters will only interfere when they are at the same level of structure as that of the 
attended letter. It is unclear what to make of the absence of interference from letters defined at the level of structure that has not been attended. Is it true that these do not get identified, or would other measures show that they have been identified?

\section{EXPERIMENT 1}

In the first study done with the present procedure (Paquet \& Merikle, 1988), two different measures of nontarget processing were used. Besides the concurrent interference measure, Paquet and Merikle also used a measure (described in more detail below) labeled the "category effect," which indicated semantic analysis for global, but not for local, ignored items. The strategy adopted in the present series of experiments was to explore selective attention in this paradigm, but to employ three different measures of semantic processing. The basic strategy was to see whether all three measures converged on a common conclusion. For example, would unattended letters in this paradigm achieve some semantic analysis at both levels? Or is semantic analysis (defined with the use of all three measures) limited to only the global level of structure for ignored items?

Evidence for extensive semantic analysis of ignored items has been reported by van der Heijden, Hagenaar, and Bloem (1984) in a task that was essentially the same as the original of Kahneman and Henik's (1981) requiring the naming of ink colors and the ignoring of irrelevant color words. It is not clear that their findings can be more generally applied to a situation in which multiple levels of structure exist in the irrelevant items, as in the present case. Indeed, Paquet and Merikle's (1988) results indicate that global and local levels of structure in ignored objects do not receive equal semantic analysis. Furthermore, van der Heijden et al. used only one measure of semantic processing, which in some instances indicated very rapid decay of semantic codes for unattended items.

In the present study, two measures of semantic analysis for ignored items were included that were identical to those reported by Paquet and Merikle (1988). The first measure was the degree of concurrent interference caused by ignored letters; this was based on whether the ignored letters indicated a response compatible or incompatible with the actual target. A second indicant of semantic processing was the category effect. Paquet and Merikle found that even when there was no evidence that global distractors (i.e., possible targets) had been identified when a local target was presented (as indicated by the absence of concurrent interference), the average response latency was slower than that in a baseline condition in which distractors were neutral letters. They speculated that this was evidence for a mandatory global categorization of all items prior to selection and suggested that subjects may simply categorize distractors as possible targets, as opposed to neutral letters, without explicitly identifying the individual letters. For the present purposes, the critical issue is whether the category of distractor letters (i.e., possible target vs. neutral letter) can affect responses to the attended letters, regardless of whether there is any effect of conflict between target and distractor identity.

To investigate this category effect in the present experiment, some trials that had only one target letter were included, with no possible target letters in the irrelevant shape. All letters in the irrelevant shape on these baseline trials were neutral and were not assigned any response. It was expected that if subjects would at least categorize the distractor letters prior to filtering them out, responses would be slower when distractors were possible targets as opposed to neutral letters.

These two measures were found by Paquet and Merikle (1988) to indicate that only the global level of structure for ignored objects received semantic analysis. When possible targets in these ignored objects were defined at the local level, attention to global targets did not result in either concurrent interference or a category effect. Irrelevant local letters were apparently filtered out with great efficiency, as long as they were not at the same level of structure as the attended letters were. One possibility, obviously, is that no semantic analysis of irrelevant letters takes place at all in this case. If this is not true, two explanations for the lack of interference effects are plausible. The irrelevant information might simply decay when not reinforced via selective attention (see, e.g., Broadbent, 1970; van der Heijden, 1981), and, as mentioned above, there is some evidence that this can take place in a different version of this paradigm (van der Heijden et al., 1984). Alternatively, evidence has suggested that ignored information is actively inhibited in selective attention (see, e.g., Neill, 1977; Tipper, 1985; Tipper \& Driver, 1988). For example, Tipper's subjects named one of two superimposed outline figures that were drawn in different colors (e.g., they could name the object drawn in red and ignore the superimposed object drawn in green). Both recall and recognition of irrelevant objects were poor, indicating good selectivity. However, a negative priming effect was observed. If the object that was ignored on trial $n$ was then used as a target on trial $n+1$, naming latency was increased. This negative priming effect also occurred when items conceptually related to that ignored on trial $n$ were used as targets on the next trial.

It is possible that such inhibition is a mechanism of selection in the current paradigm. Experiment 1 was an attempt to determine whether negative priming would be observed for stimuli that appeared to have been ignored. If information that does not cause concurrent interference (or a category effect) on one trial is nevertheless inhibited on the next trial, this would indicate that letters at irrelevant levels of structure in this task were inhibited and not filtered before higher level codes could be activated. Negative priming thus represents the third indicant of semantic processing, which would reflect inhibition of ignored letters. Responses to targets that had just appeared as irrelevant letters (incompatible with the target) on the previous trial were compared with responses on the baseline trials described above. 
In addition to the three different indicants of semantic analysis for ignored items in this paradigm, procedures were also used in order to vary the difficulty of selecting the relevant object. Paquet and Merikle (1988) showed that both concurrent interference and the category effect were reduced, but not eliminated, by making selection easier (see also Paquet, 1988), whereas Briand and Klein (1993) have found that interference was totally eliminated by some manipulations. An additional goal of the present study was to determine whether such procedures would have equivalent effects on all three of the measures used in the present experiment, and Experiments 2 and 3 were included with this goal in mind.

\section{Method}

Subjects. Eight Dalhousie University undergraduates participated for payment; all had normal or corrected vision, according to their self-reports.

Apparatus and Procedure. The stimuli were shown on a Tektronix 604 oscilloscope, controlled by a PDP-11/03 computer. The computer controlled presentation of stimuli, feedback, and response collection. The subjects responded by pressing one of two keys on a response board placed on a table in front of them. Their right and left index fingers were used for right and left keys, respectively. The subjects placed their heads on a chinrest at the edge of the table. The viewing screen was $72.8 \mathrm{~cm}$ away, with a fixation point at the center. A circle and a square appeared on either side of this fixation, with the location of specific shapes varying randomly from trial to trial.

The horizontal distance from the fixation point to the nearest edge of each shape was $10^{\circ}$. The square and circle were approximately $2.5^{\circ} \times 2.5^{\circ}$ in size. The shapes were plotted as a series of points, not continuous lines, and the density of the lines was 14 points/deg. At the viewing distance and duration used, the lines appeared continuous. Compound letter patterns were centered in each of the shapes and were based on a $5 \times 7$ matrix. The size ratio of global letters to local ones was $10: 1$. Global letters were $1.19^{\circ}$ wide $\times$ $1.42^{\circ}$ high, whereas local letters were $.11^{\circ} \times .13^{\circ}$. Local letters were separated horizontally by $.16^{\circ}$ and vertically by $.09^{\circ}$.

The subjects performed two blocks of trials on separate days, in sessions lasting approximately $1 \mathrm{~h}$ each. In one session, they responded to global targets, and in the other session, they responded to local targets; the order in which these sessions were given was counterbalanced. The target letter appeared in a predesignated shape at the same level of structure within a session. The target letters were $\mathrm{T}$ and $\mathrm{H}$; the neutral letters were $\mathrm{L}$ and $\mathrm{E}$. These particular stimuli were picked to reduce the chance that the subjects could discriminate between the target letters on the basis of some preattentively available attribute. Examples of the stimuli are shown in Figure 1.

The subjects were tested in a darkened room with their heads resting in a chinrest adjusted to a comfortable height. The relevant shape was either a circle or a square (counterbalanced between subjects), and it remained constant for both sessions. The subjects initiated a block of trials by pressing either response key. On each trial, the fixation point appeared $1 \mathrm{sec}$ before the stimulus display. The stimulus pattern was shown for $180 \mathrm{msec}$ to minimize the opportunity for eye movements to the attended location, following which the fixation point remained on. The subjects decided whether the target letter in the relevant shape was $\mathrm{T}$ or $\mathbf{H}$ and pressed the appropriate response key (counterbalanced between subjects). Both speed and accuracy were stressed. Following a response, the subjects received feedback only if they had made an error. Responses that took longer than $1.8 \mathrm{sec}$ were considered to be errors. Rest periods were given after every 20 trials, and the subjects pressed a response key to initiate another set of 20 trials.

Because of the need to look at sequential effects, random trial selection was not used. Instead, the subjects went through a fixed sequence of 437 trials twice, once for global and once for local targets. In all there were 15 different conditions, which will be described below.

Concurrent trials. These trials are referred to here as concurrent because two of the letters shown were possible target letters. Only one of these was to be attended, with the other being designated as an ignored target. Concurrent interference was based on comparing responses to targets paired with ignored targets that were either the same as or different from the target letter. In each stimulus display, there were two possible target letters and two neutral ones. One target letter was in the relevant shape at the predesignated level of structure. The ignored target letter could be at the opposite level of structure in the relevant shape or at either level of structure in the irrelevant shape. The two neutral letters were used as needed, to create two compound letters on each trial. For example, if the target was $\mathrm{T}$ and was defined at the global level, the compound pattern might be a large $T$ made of small Ls. If the target was defined locally, the subjects would see a large $\mathrm{L}$ made of small Ts. The ignored target letter's name could of course be compatible or incompatible with the response required to the target, resulting in 3 (ignored-letter location) $\times 2$ (compatible or incompatible) $=6$ possible conditions. Each of these conditions had 32 trials per subject, in such a way that each particular target was paired with each particular ignored target and combination of neutral letters equally often.

One-target trials. Nine conditions had only one target letter per trial, which was shown at the appropriate level in the relevant shape. The other three letters present were all neutral. Each of the onetarget conditions involved 24 trials per subject. Trials from six of these conditions always followed one of the concurrent trials and contained a target letter that had been the ignored target on the previous trial (note that when following compatible trials, this letter would in fact be the same as the target on the previous trial). Each possible target letter was shown equally often, as was each combination of the neutral letters. ${ }^{1}$

Two other one-target conditions contained the target letter from the previous concurrent trial. These trials were drawn equally from instances in which the previous trial had included a compatible or an incompatible distractor (the identity and level of the distractor letter was ignored in data analyses). These conditions were included so that on one-target trials, the subjects were not always responding to the ignored target from the previous concurrent trial.

Baseline trials. Baseline trials will be distinguished from other one-target trials. A baseline trial contained only one target letter, which could not have occurred on the previous trial. All baseline trials followed other one-target trials and thus were alternations (i.e., Target $\mathrm{T}$ followed by Target $\mathrm{H}$ ). Each target letter was shown equally often, as was each combination of neutral letters.

Fillers. The total of all concurrent, one-target, and baseline trials was 408 . This was augmented by 29 filler trials, which were used to break up sequences in which the same target came up too often. Filler trials were always one-target trials, and the target was different from that of the previous and subsequent trials. The first 10 trials of each session were filler trials, but the order of these was random.

At the start of each session, practice was given until the experimenter was satisfied that the subject understood the task and was performing reasonably well. This typically took $20-40$ trials.

\section{Results}

In this and subsequent experiments, the RTs (reaction times) and error rates were included in analyses of variance (ANOVAs) with target level and condition (15 levels) 
a

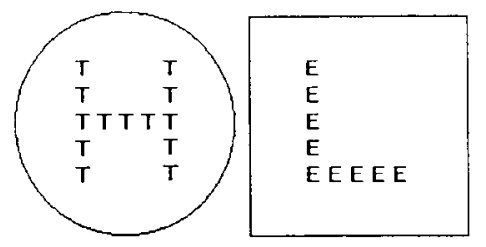

$\mathrm{b}$

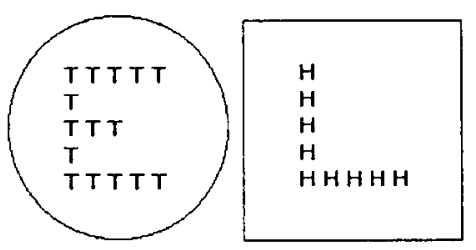

c

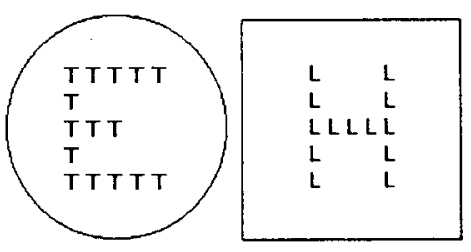

d

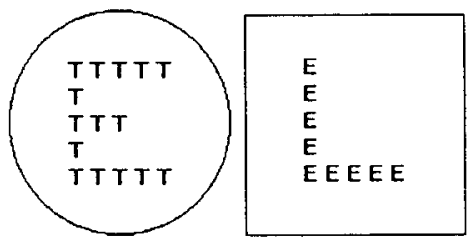

e

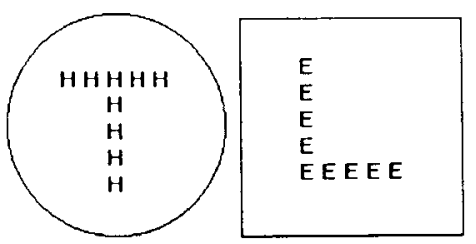

f

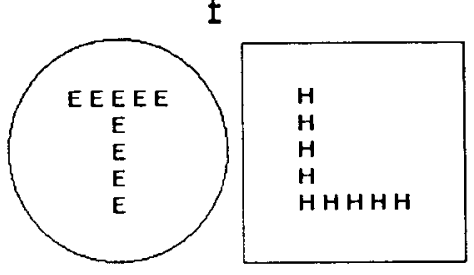

9

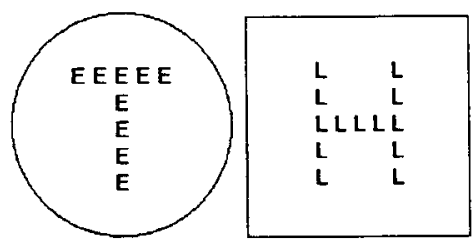

$\mathrm{h}$

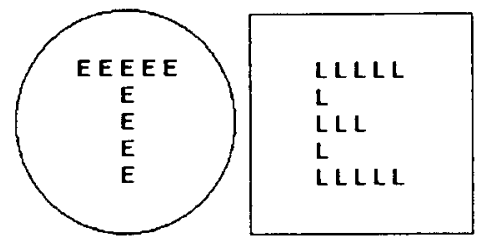

Figure 1. Examples of stimuli used in the three experiments. In the examples given, the relevant pattern is defined as the one within the circle, the target letter is $T$, and the ignored target (when present) is the letter H. Stimuli a-d illustrate local targets, whereas e-h illustrate global targets. The ignored-target distractor is within the circle $(a, e)$, defined locally (b, f) or globally (c, g) within the square, or absent $(d, h)$.

as factors. A pooled mean square error $\left(M S_{\mathrm{e}}\right)$ was then calculated by combining the error rates for the condition and target level $\times$ condition effects. This was then used to evaluate the relevant planned contrasts. To facilitate comparison of the three measures used, the summary tables of results present only the difference scores for the particular comparisons. Furthermore, since there are a large number of comparisons to consider, the summary tables are used to indicate the significance levels of the effects. Appendixes A and B are provided for readers who wish to see mean values of the actual data on which these comparisons are based. Preliminary analyses of the data from Experiment 1 revealed a significant effect of target level in $\mathrm{RT}[F(1,7)=31.89, p<.001$; RTs were 627 and $799 \mathrm{msec}$ for global and local targets, respectively]. There was also a significant effect in error rate $[F(1,7)=$ $12.32, p<.01$; error rates were $5.1 \%$ and $13.0 \%$ for global and local targets]. The pooled error terms for the RT and error rate comparisons were 1,320.8 and 35.8, respectively $(d f=1,196)$.

Concurrent interference. The results from concurrent trials can be summarized as follows. Interobject compatibility effects followed the pattern observed by Paquet and Merikle (1988). That is, the identity of ignored targets only mattered when these were at the same level of structure as the actual target. Intraobject compatibility effects showed that global distractors interfered with responses to local targets but that local distractors failed to interfere with global targets (see Table 1).

The interobject interference was significant when the target and ignored target were at the same level of structure, and this was true for both RT and error rate $(p<$ .001 in all cases). There were no significant effects in either RT or error rate when target and ignored target 
Table 1

Experiment 1, Shape Cues: Concurrent Interference, Negative Priming, and the Category Effect in Reaction Time (RT) and Percent Errors (\%E), as a Function of Target Level and Ignored-Target Location

\begin{tabular}{|c|c|c|c|c|c|c|}
\hline \multirow[b]{4}{*}{ Targets } & \multicolumn{6}{|c|}{ Location of Ignored Target } \\
\hline & \multirow{2}{*}{\multicolumn{2}{|c|}{ On Attended }} & \multicolumn{4}{|c|}{ On Unattended } \\
\hline & & & \multicolumn{2}{|c|}{$\begin{array}{l}\text { Ignored, } \\
\text { Local } \\
\end{array}$} & \multicolumn{2}{|c|}{$\begin{array}{c}\text { Ignored, } \\
\text { Global }\end{array}$} \\
\hline & RT & $\% \mathrm{E}$ & RT & $\% \mathrm{E}$ & RT & $\% \mathrm{E}$ \\
\hline \multicolumn{7}{|l|}{ Local } \\
\hline Concurrent interference & $39 \ddagger$ & 5.1 & $72^{*}$ & $20 *$ & 23 & -4.3 \\
\hline Negative priming & 11 & 4.5 & 22 & $6.5 \ddagger$ & $46 \ddagger$ & $8.1 \dagger$ \\
\hline Category effect & 25 & 4.9 & -3 & $14.3^{*}$ & $69^{*}$ & $7.7 \dagger$ \\
\hline \multicolumn{7}{|l|}{ Global } \\
\hline Concurrent interference & -7 & 0.0 & -22 & -2.4 & $72 *$ & $11.3 *$ \\
\hline Negative priming & 12 & -2.2 & 29 & 3.0 & 25 & 3.6 \\
\hline Category effect & 20 & .7 & 22 & 1.9 & $64 *$ & 9.1 * \\
\hline
\end{tabular}

${ }^{*} p<.001 . \dagger p<.01 . \ddagger p<.05$.

were at different levels of structure (all $F \mathrm{~s}<2$ ). As Table 1 shows, intraobject effects are all but nonexistent when global targets are paired with local ignored targets. When local targets are paired with global ignored targets, there is a significant compatibility effect in RT and a strong trend in error rate.

Negative priming. The measure of negative priming was based on trials on which a particular target on a onetarget trial had been an ignored target inconsistent with the target letter on the previous concurrent trial. Performance on such trials was compared with that on the baseline trials. A significant difference in RT (or accuracy) is compatible with the interpretation that those letters had been inhibited during the concurrent trial in order to allow efficient target processing. As it turned out, negative priming was only obtained when the target was local and the previous ignored target had been within the irrelevant object (see Table 1).

When the target was local, and the ignored target was global, negative priming had a significant effect on both RT $(p<.05)$ and error rate $(p<.01)$. When the ignored target had been defined at the local level of structure, negative priming was significant only in error rate $(p<.05)$. The latter effect is not particularly interesting, since the same condition shows evidence for semantic analysis of the ignored targets using concurrent interference. Of particular interest for the present purposes is whether any of the three measures indicates semantic analysis for ignored items. The negative priming effect from global ignored targets is more critical, because the concurrent interference measure suggested that those letters had received little or no semantic analysis.

Category effects. As described earlier, the category effect is based on comparing performance on concurrent trials (collapsed across ignored-target compatibility) with performance on the baseline or neutral trials. Category effects will be considered for three different cases. In the first, and most interesting, the ignored target is in the irrelevant object and at the level of structure opposite to that of the target. Note that these two conditions showed no evidence for a concurrent interference (see Table 1). When global ignored targets were paired with local targets, the category effect was significant in both RT and error rate. However, when local ignored targets were paired with global targets, neither RT nor error rate showed a category effect.

Two conditions are less interesting in the present context-namely, those with the target and ignored target defined at the same level of structure. As Table 1 indicates, there is evidence for a category effect in these cases, but since the same two conditions show large concurrent interference effects, it is obvious that these ignored targets received extensive semantic analysis. Finally, as Table 1 makes clear, category effects were not obtained in a single instance when the ignored target was contained within the relevant object. It is particularly compelling that when the target was global, local ignored targets did not show any evidence for semantic analysis according to any of the measures, whether they were in the ignored object or contained within the object that was the focus of attention.

\section{Discussion}

The results from the concurrent trials are consistent with previous findings (Paquet \& Merikle, 1988). Concurrent interference from ignored targets appearing in an irrelevant object occurs only when these are at the same level of structure as the target letter. Furthermore, when the ignored targets were in the same object as the target letters were, global precedence was found. Global distractors interfered with local targets, but the opposite was not true. The occurrence of both negative priming and the category effect also suggests some form of global precedence.

As for negative priming effects, consider the case in which the target is local and the ignored targets are at the global level of the irrelevant object. The interference measure from trial $n$ (concurrent trials) indicates that these ignored targets did not affect responses to targets. How- 
ever, on trial $n+1$ (one-target trials), negative priming resulted. It seems, therefore, that when the subjects identified local targets, information contained at the global level was filtered out via inhibition of encoded representations resulting from that information.

With global targets, a different picture emerges. When the ignored target on trial $n$ is at the local level of the irrelevant object, no concurrent interference was found. However, in contrast to the results mentioned above, there was no negative priming on trial $n+1$. Apparently, the information provided by the local level on trial $n$ was filtered without inhibition of encoded representations.

The category effect showed that when target letters were defined at the local level, the subjects were slow and less accurate (relative to a baseline) when the unattended global letters in the irrelevant object were possible target letters. However, no similar decrement in performance was observed when target letters were global and the unattended distractors were local. A working hypothesis is that the factors that lead to processing of global ignored targets vis-à-vis the category effect are the same as those that caused negative priming to be observed only for global ignored targets. According to this view, global information would appear to be more difficult to filter out than would local information (at least for the present stimuli; see Kinchla \& Wolfe, 1979), perhaps because of a mandatory preattentive global analysis of visual information (see Paquet \& Merikle, 1988).

\section{EXPERIMENT 2}

Briand and Klein (1993) demonstrated that when one compares the cuing attributes of form and color in the context of this paradigm, color cues are more effective. Interobject interference is greatly reduced or eliminated. However, this demonstration of efficient selection with color as the relevant attribute involved only one measure of semantic processing for ignored letters-namely, concurrent interference. In Experiment 2, the same procedure as that in Experiment 1 was used to measure concurrent interference, negative priming, and the category effect. However, color was now the attribute defining target location. It was expected that concurrent interference would decrease if color should act as a more efficient selection cue. Of particular interest was whether the additional indicants of semantic processing would also show evidence for better selectivity.

\footnotetext{
Method

Subjects. Fourteen Dalhousie undergraduates participated for pay; all had normal or corrected vision.

Apparatus and Procedure. The stimuli were shown on a color TV monitor controlled by an Apple II + computer. A fixation patch (approximately $.10^{\circ}$ square) was displayed at the center of the screen. Global letters were created from a $5 \times 7$ matrix; the global letters were $1.38^{\circ}$ wide $\times 2.70^{\circ}$ high. The local letters making up this pattern were $.21^{\circ} \times .34^{\circ}$. On each trial, two compound letters were presented, one red and the other blue. The nearest edge of each compound letter was $.88^{\circ}$ left or right of fixation. All subjects received the same sequence of trials as that used in Experi-
}

ment 1 ; the color of the letters replaced the shape of a surrounding outline as the attribute used to select targets. (See Experiment 1 for details of the makeup of the 437 trials.) The subjects responded to global and local targets in two sessions given on different days, and the order of these, as well as which color had to be attended to, was counterbalanced across subjects. Target letters were $T$ and $H$, and neutral letters were $L$ and $E$.

On a trial, the sequence was as follows. A fixation patch appeared for $1.5 \mathrm{sec}$, following which the compound letters were shown for $183 \mathrm{msec}$. The subjects were required to respond via the computer keyboard whether a $\mathrm{T}$ or an $\mathrm{H}$ was shown. The 0 and 1 keys were used, and this was varied between subjects. Responses slower than $1.8 \mathrm{sec}$ were assumed to be errors. A 1 -sec blank screen preceded the appearance of the fixation patch to start the next trial.

\section{Results}

Preliminary analyses of RT and error rate data were carried out as described previously. There was a main effect of target level on RT $[F(1,13)=20.62, p<.001$; 570 vs. $643 \mathrm{msec}$, global and local, respectively] but not on accuracy $(F<1 ; 3.9 \%$ and $3.6 \%$ errors). The pooled $M S_{\mathrm{e}} \mathrm{s}$ were $1,266.8$ and 12.5 for RT and error rate, respectively $(d f=1,364)$. The summarized data are presented in Table 2, and the original values are presented in Appendixes $\mathrm{A}$ and $\mathrm{B}$.

Concurrent interference. When the ignored target was in the irrelevant object, there was no interference with responses to local targets (see Table 2). In particular, the interference from local ignored targets was virtually nonexistent. This is striking, given the difficulty reported by Paquet and Merikle (1988; see also Paquet, 1988) in eliminating interference in this case. When the ignored target was global, there was a significant effect on RT, but this was actually opposite to that expected, indicating facilitation, if anything $(p<.05)$. Ignored targets interfered with local targets only when they were global and located within the relevant object $(p<.001)$.

With global targets, none of the interference effects were significant, although there was an effect on RT that was just short of significance $[F(1,364)=3.83, p<.06]$ when the ignored targets were global.

Negative priming. Only one condition showed significant evidence for negative priming (global targets, global ignored targets). However, caution should be used in interpreting this result because it went along with an opposite trend in error rate, indicating the possibility of a speed-accuracy tradeoff. The only other significant effect came with global targets and ignored targets (local) within the relevant object. However, as Table 2 makes clear, this did not indicate inhibition but rather facilitation and thus is difficult to interpret.

Category effects. When the target was local, there were significant category effects when the ignored target was global. When the ignored target was contained within the relevant object this was less meaningful because the concurrent interference measure also indicated semantic analysis of those letters. However, the significant category effect for global ignored targets contained within the irrelevant object represents the only evidence that those letters were identified when the target was local (i.e., there 
Table 2

Experiment 2, Color Cues: Concurrent Interference, Negative Priming, and the Category Effect in Reaction Time (RT) and Percent Errors (\%E), as a Function of Target Level and Ignored-Target Location

\begin{tabular}{|c|c|c|c|c|c|c|}
\hline \multirow[b]{4}{*}{ Targets } & \multicolumn{6}{|c|}{ Location of Ignored Target } \\
\hline & \multirow{2}{*}{\multicolumn{2}{|c|}{ On Attended }} & \multicolumn{4}{|c|}{ On Unattended } \\
\hline & & & \multicolumn{2}{|c|}{$\begin{array}{l}\text { Ignored, } \\
\text { Local }\end{array}$} & \multicolumn{2}{|c|}{$\begin{array}{c}\text { Ignored, } \\
\text { Global }\end{array}$} \\
\hline & RT & $\% \mathrm{E}$ & RT & $\% \mathrm{E}$ & RT & $\% \mathrm{E}$ \\
\hline \multicolumn{7}{|l|}{ Local } \\
\hline Concurrent interference & $46^{*}$ & 2.5 & 7 & -.8 & $-29 \dagger$ & -1.4 \\
\hline Negative priming & 10 & -.2 & 26 & 1.3 & 26 & .3 \\
\hline Category effect & $28 \dagger$ & 1.5 & 14 & 1.2 & $46^{*}$ & $2.3 \dagger$ \\
\hline \multicolumn{7}{|l|}{ Global } \\
\hline Concurrent interference & 25 & 1.6 & -9 & -.2 & 27 & 1.2 \\
\hline Negative priming & -1 & $-3.2 \dagger$ & 16 & -1.9 & $28 \dagger$ & -1.8 \\
\hline Category effect & 21 & -.5 & 17 & -2.3 & 24 & -.5 \\
\hline
\end{tabular}

was no concurrent interference and only weak evidence for negative priming). When the target was global, none of the category effects were significant.

\section{Discussion}

When color is used as the selection cue, the interobject effects are not the same as they are when form is used as a cue. There is no significant concurrent interference from ignored targets at the same level of structure as that of the target. If there is concurrent interference in this situation, it appears to be limited to the case when both the target and the ignored target are global. As was the case in Experiment 1, global ignored targets interfered with local targets when displayed inside the same shape, but local ignored targets had no significant effect on global targets. Thus, color cues appeared to make it easier to select the relevant object but had no effect on within-object interference effects.

Further evidence of efficient filtering by color came from the finding that there was only weak evidence for negative priming in Experiment 2. The one condition that showed this effect in Experiment 1 (local targets with global ignored targets in the irrelevant shape) showed only marginal evidence for inhibition of distractors in the present experiment, despite the fact that the present experiment should have had more power, given the larger sample size used. It was suggested following Experiment 1 that there was a common basis for the negative priming and category effects, since both derived from the same condition (global ignored targets paired with local targets). This possibility is questionable, given the results of Experiment 2. Global ignored targets in the irrelevant object (with local targets) resulted neither in concurrent interference on the trial on which they were shown nor in inhibition when they were used as targets on the next trial. Yet there was still a significant category effect for these global distractors. Some caution should be used on this point, given that the inhibition data indicate a marginal effect on RT $(26 \mathrm{msec}, p<.08)$.
In summary, cuing by color seemed to allow more efficient selection, to the extent that ignored targets shown in an unattended object (1) do not interfere with performance on the trial on which they are shown and (2) need not be inhibited in order to allow targets to be responded to efficiently. Despite this, there is evidence that either a mandatory global categorization or mandatory global identification does occur for all items in the display, regardless of whether form or color is used to select targets. Subjects appear able to ignore this information when responding to specific targets.

\section{EXPERIMENT 3}

Paquet and Merikle (1988) found that when a spatial cue such as an arrow was used to direct attention to the relevant target pattern in this paradigm, both interobject interference and the category effect were reduced but still significant. This was the case for a stimulus onset asynchrony (SOA) of $100 \mathrm{msec}$ (Paquet \& Merikle, 1988). Since cuing intervals this short may not allow for full allocation of attention to the cued location (Muller \& Findlay, 1988; Muller \& Rabbitt, 1989; Remington \& Pierce, 1984), any apparent semantic analysis of the irrelevant items might have been due merely to a failure to restrict attention to only the relevant location.

Briand (1989) has obtained evidence that at longer SOAs $(183$ or $600 \mathrm{msec}$ ), interobject interference is completely eliminated in this paradigm. Cuing intervals as long as this should allow spatial attention to be completely focused on the relevant location. However, that study involved only one measure of semantic processing-namely, the amount of concurrent interference caused by ignored targets. Other measures might have revealed extensive semantic analysis. In this final experiment, the subjects received a spatial cue indicating the target location $600 \mathrm{msec}$ before the display items appeared. It was expected that interobject interference effects would be eliminated, but it was unclear what would be observed with 
either the negative priming or the category effect measures. In the case of negative priming, for example, it was possible that distractors would not be fully processed and thus would not need to be inhibited. The category effect, however, has been shown to occur somewhat independently of negative priming (see Experiment 2) and might still be observed even if negative priming was not found.

\section{Method}

Subjects. Twelve Dalhousie undergraduates participated for extra course credit. All had normal or corrected vision.

Apparatus and Procedure. This experiment was carried out with the use of the PDP-11/03 and oscilloscope system employed in Experiment 1 . The procedure was identical to that in Experiment 1, except for a change in the type of cue. In the present experiment, form was now irrelevant as a cue to target location. The subjects initiated each trial by pressing a footpedal. One second after this, an arrow appeared at the fixation point, and $600 \mathrm{msec}$ later a display identical to that used in Experiment 1 was shown for $180 \mathrm{msec}$. The subjects were required to report only the global (or local) letter that was within the shape pointed to by the arrow and to ignore whatever was in the other shape. The particular shape surrounding the target pattern was determined randomly on each trial, and the alternative shape surrounded the nontarget pattern. The subjects received an error if no response was made within $1.8 \mathrm{sec}$. Target letters were $\mathrm{T}$ and $\mathrm{H}$, whereas distractors were $\mathrm{L}$ and $\mathrm{E}$.

The subjects in Experiment 3 wore an eye movement monitor (Eye-Trac Model 210). This was calibrated to the left eye, and a sample was read every $30 \mathrm{msec}$ (screen refresh rate). If a discrepancy in eye position of greater than $.40^{\circ}$ was observed from a point just prior to onset of the arrow cue up to any point prior to response, that trial was suspended. The subjects received a tone as feedback, and the results from that trial were not used in data analysis. Furthermore, if that trial was one member of a twoletter-one-letter pair used to assess negative priming, the results from both of those trials were excluded from analyses.

\section{Results}

On the average, eye movements were detected on only 10.8 out of 437 trials (11.8 and 9.8 for global and local target conditions, respectively). Preliminary analyses indicated significant effects of target level on both RT
$[F(1,11)=25.79, p<.001 ; 478$ vs. $578 \mathrm{msec}$, global and local, respectively $]$ and error rate $[F(1,11)=14.63$, $p<.01 ; 2.1 \%$ vs. $5.3 \%$ ]. The $M S_{\mathrm{e}}$ s for the RT and error rate contrasts were 921.2 and 16.4 , respectively $(d f=$ $1,308)$. Table 3 presents a summary of the effects of interest; the original data are presented in Appendixes $A$ and $B$.

Concurrent interference. Planned contrasts showed that when the ignored target was in the irrelevant object, there was no interference in any case (see Table 3). However, when the ignored target was within the relevant object, interference followed the pattern shown in Experiments 1 and 2. Namely, global ignored targets interfered with responses to local targets $(p<.01)$, but local ignored targets did not interfere with global targets.

Negative priming. When the ignored target on trial $n$ had been in the irrelevant object, there was only one finding indicative of negative priming on trial $n+1$. This was when the target was local and the ignored target had been global ( $p<.05$ for error rate). Negative priming from ignored targets within the relevant object were either in the wrong direction (global targets) or redundant because concurrent interference already indicated semantic analysis of ignored targets (local targets; see Table 3).

Category effects. When the target was local, there was evidence for category effects for ignored targets in all three possible locations. When the ignored target was within the irrelevant object, category effects were limited to error rate $(p<.01)$, although an effect was obtained in both error rate and RT for ignored targets within the relevant object. For global targets, only global ignored targets within the irrelevant object resulted in a category effect $(p<.01$ for RT).

Validity of negative priming measure. The measure of negative priming used in these experiments might be open to criticism on the following grounds. The comparison of interest is that between one-target trials (which always follow concurrent trials) and baseline trials (which

Table 3

Experiment 3, Location Cues: Concurrent Interference, Negative Priming, and the Category Effect in Reaction Time (RT) and Percent Errors (\%E), as a Function of Target Level and Ignored-Target Location

\begin{tabular}{|c|c|c|c|c|c|c|}
\hline \multirow[b]{4}{*}{ Targets } & \multicolumn{6}{|c|}{ Location of Ignored Target } \\
\hline & \multirow{2}{*}{\multicolumn{2}{|c|}{ On Attended }} & \multicolumn{4}{|c|}{ On Unattended } \\
\hline & & & \multicolumn{2}{|c|}{$\begin{array}{l}\text { Ignored, } \\
\text { Local }\end{array}$} & \multicolumn{2}{|c|}{$\begin{array}{c}\text { Ignored, } \\
\text { Global }\end{array}$} \\
\hline & RT & $\% \mathrm{E}$ & RT & $\% \mathrm{E}$ & RT & $\% \mathrm{E}$ \\
\hline \multicolumn{7}{|l|}{ Local } \\
\hline Concurrent interference & $84 *$ & $12.5^{*}$ & -1 & 1.0 & -14 & -1.0 \\
\hline Negative priming & 5 & $3.5 \dagger$ & -11 & 1.9 & 3 & $3.5 \ddagger$ \\
\hline Category effect & $30 \dagger$ & $8.3^{*}$ & 1 & $3.7 \dagger$ & 17 & $4.0 \dagger$ \\
\hline \multicolumn{7}{|l|}{ Global } \\
\hline Concurrent interference & -1 & -.9 & 1 & -1.5 & 15 & .7 \\
\hline Negative priming & $-24 \ddagger$ & -.3 & -9 & .2 & -1 & -.5 \\
\hline Category effect & 4 & .8 & -6 & .7 & $29+$ & 1.1 \\
\hline
\end{tabular}

${ }^{*} p<.001 . \quad \dagger p<.01 . \ddagger p<.05$. 
always follow one-target trials). One would expect that all other things being equal, the concurrent trials should be the most difficult for subjects, because such trials always involve two potential targets. In fact they might be so difficult that they cause subjects to respond more slowly on subsequent trials, in a manner analogous but not equivalent to the psychological refractory period. If this is the case, it should be obvious from the description above that one-target trials, but not baseline trials, would be affected by this temporary decrement in performance. Thus if onetarget trials result in slower RTs than baseline trials do, this may not be due to negative priming but may instead be an artifact. ${ }^{2}$

To determine whether this actually did affect the negative priming measures, the following analysis was performed. First, for each subject, the conditions with the slowest and fastest mean RT were determined for concurrent trials. This was done separately for trials with global targets and those with local targets, and also for trials with compatible and those with incompatible distractors. Then, their mean RTs in the corresponding one-target trials that followed these slow (and fast) conditions were recorded. This was done for each subject and experiment, and the data were included in an ANOVA with experiment, compatibility, global versus local target, and fast versus slow responses as factors. The explanation offered in the preceding paragraph leads to the following prediction.

Since concurrent trials might be relatively difficult, they might cause a decrement in performance on subsequent one-target trials if subjects are temporarily ill-prepared to deal with those stimuli. This suggests that for a given subject, concurrent trials that lead to the slowest responses should result in a large decrement on subsequent onetarget trials. Conversely, concurrent trials that result in relatively fast responses should not cause much of a decrement on subsequent one-target trials. Therefore, by comparing one-target trials that follow either slow or fast responses, one can assess whether this potential artifact actually has affected performance. If it has, subjects should be slower and less accurate on one-target trials that follow slow concurrent trials than they are on one-target trials that follow fast concurrent trials.

The results of the analysis of one-target trials following either slow or fast concurrent trials is presented in Table 4. This includes the actual performance on the concurrent trials for purposes of comparison, although the analysis of the data was based only on the corresponding one-target trials that followed these. It should be clear that performance on one-target trials is not affected by the difficulty of the preceding concurrent trial; analyses of RT and accuracy did not reveal significant effects of this variable (both $F$ s $<1$ ), nor was any interaction involving it significant. The only factors affecting performance on the one-target trials were experiment [for RT, $F(2,31)=$ $11.36, p<.001$; for error rate, $F(2,31)=5.98, p<$ $.01]$, and whether the target was local or global [for RT, $F(1,31)=74.85, p<.001$; for error rate, $F(1,31)=$ $11.13, p<.01] .{ }^{3}$ Thus it does not appear that the artifact
Table 4

Mean Reaction Time (RT) and Percent Errors (\%E) for Fastest and Slowest Trials on trial $n$ (Concurrent Trials) and

Subsequent Performance on trial $n+1$ (One-Target Trials)

\begin{tabular}{|c|c|c|c|c|c|c|c|c|}
\hline \multirow[b]{4}{*}{ Experiment } & \multicolumn{8}{|c|}{ Trials } \\
\hline & \multicolumn{4}{|c|}{ Slow } & \multicolumn{4}{|c|}{ Fast } \\
\hline & \multicolumn{2}{|c|}{ trial $n$} & \multicolumn{2}{|c|}{ trial $n+1$} & \multicolumn{2}{|c|}{ trial $n$} & \multicolumn{2}{|c|}{ trial $n+1$} \\
\hline & RT & $\% \mathrm{E}$ & RT & $\% \mathrm{E}$ & RT & $\% \mathrm{E}$ & $\mathrm{RT}$ & $\% \mathrm{E}$ \\
\hline 1 & 753 & 18.6 & 710 & 8.2 & 700 & 5.9 & 706 & 7.2 \\
\hline 2 & 645 & 6.1 & 608 & 3.2 & 584 & 2.8 & 602 & 3.2 \\
\hline 3 & 564 & 8.1 & 525 & 2.8 & 512 & 2.0 & 524 & 4.0 \\
\hline$M$ & 642 & 9.7 & 603 & 4.2 & 586 & 3.2 & 599 & 4.4 \\
\hline
\end{tabular}

under consideration here affected performance on onetarget trials.

\section{Discussion}

When the location of the target letter is cued in advance, concurrent interference from ignored targets in an irrelevant object is reduced but is still significant in this paradigm (Paquet, 1988; Paquet \& Merikle, 1988). The present results show that interference is in fact totally eliminated when subjects have sufficient time (e.g., $600 \mathrm{msec}$ ) to orient attention to the cued location prior to target onset. Despite this, there is still evidence for semantic analysis of ignored targets. First, global ignored targets lead to both negative priming and a category effect when the target is local. Second, local ignored targets lead to a category effect when the target is local. Finally, global ignored targets lead to a category effect when the target is global. The simplest way to summarize these results is to state that global ignored targets (in an irrelevant object) receive semantic analysis regardless of whether the target is global or local, whereas local ignored targets receive semantic analysis only when the target is local. This is the same conclusion reached by Paquet and Merikle (1988). However, the present results are somewhat more compelling than those of Paquet and Merikle (1988), because they show that this pattern is obtained even when attention is focused on the target location efficiently enough to completely eliminate concurrent interference.

Of course, this pattern is restricted to interference from ignored targets within the irrelevant object; interference from ignored targets within the relevant object was unaffected by prior allocation of attention. Indeed the results here are very clear. Global ignored targets showed evidence of semantic analysis when all three measures were used, but local ignored targets showed no evidence for semantic analysis on any of the measures.

\section{GENERAL DISCUSSION}

In previous studies done with the paradigm in question (Briand, 1993; Briand \& Klein, 1993; Paquet \& Merikle, 1988) only one, or at most two, separate measures have been used to estimate the degree of semantic analysis for unattended stimuli. The present study included three measures, with the goals of determining (1) whether 
different methods of cuing target location would have similar effects on all three measures, and (2) whether the three measures themselves would give consistent results in any particular case. It was found that the various attributes used to direct attention allowed targets to be selected with varying degrees of efficiency, but the three measures did allow some common trends to be noted.

\section{Intraobject Effects}

Before we address the interobject effects, we must consider the intraobject effects. The first point to be made is that the type of spatial cue used had little effect on the intraobject effects obtained. All three experiments revealed the typical finding that global distractors interfere with local targets, but that local distractors do not interfere with global targets. There is little evidence for negative priming, and this evidence is restricted to the local target-global distractor case, which obviously involves semantic analysis of distractors on the basis of the concurrent interference data. Finally, category effects were generally not observed for intraobject distractors, but where there was evidence for this it occurred only for global distractors. Given the large effects of concurrent interference generated by these, it is likely that what appears to be a category effect can actually be attributed to the concurrent interference that was generated.

\section{Interobject Effects}

As has been indicated, the interobject effects are of primary interest in this paradigm. To summarize briefly, the three measures appeared to be partially but not totally independent of one another. Furthermore, they were not all equally affected by the type of spatial cue used.

When concurrent interference was the measure of nontarget processing, results confirmed that form selection leads to interference exclusively from distractors at the same level of structure as that of the target. When the color of the letter patterns is used as the selection cue, or when a spatial precue is used with a long SOA, no interference whatsoever is observed. Negative priming is found when form is the selection cue, but only when targets are local and ignored targets are global. With global targets and local ignored targets, there is no evidence for negative priming, suggesting that the latter do not need to be inhibited in this case. There is weaker evidence for negative priming when color or spatial cues are used, and in fact this failed to reach significance in Experiment 2.

Finally, robust category effects are observed for global ignored targets when both form and color are used to indicate target location. There is a reduced but still significant category effect when spatial cues are used. The appearance of this category effect in all three experiments is consistent with often suggested notions that preattentive analysis is limited to a rough global analysis of scenes (Hoffman, 1975; Navon, 1977; Neisser, 1967). An initial selection of objects defined only at the global level of structure might represent a default procedure that serves to parse the visual array into a manageable number of meaningful entities. This type of analysis might be very difficult to overcome when the task is filtering-that is, when subjects must select one item from among others on the basis of some stimulus attribute with location uncertain. However, if a parallel global analysis of visual information did occur automatically, it could even suffice to disrupt target processing when subjects know in advance where the target item will appear (Experiment 3). Thus, even when subjects have had sufficient time $(600 \mathrm{msec})$ to orient attention to the cued location in advance of a target that will appear there, some mandatory preattentive global analysis of items in uncued locations appears to take place. It is particularly important that this global category effect occurred in a situation where most models of visual orienting would assume that attention was restricted to only the cued location. This strongly suggests that semantic analysis of at least the global level of structure is an unlimited capacity process. Note that Paquet and Merikle (1988), in showing evidence for mandatory global analysis, either used a filtering paradigm or cued the target location with a very brief SOA. In the first case, there is little control over where attention was directed, and it is most likely that at least some attention to irrelevant locations was allowed until subjects could tell (by virtue of the shape of the form at that location) that it was irrelevant. In the latter case, as mentioned above, spatial attention is not likely to be completely focused on the relevant location. The present results show that semantic analysis for (ignored) global information takes place even under conditions that would seem to be far more favorable to selective processing.

Table 5 summarizes the significant effects observed in the three experiments included here. For the interobject effects, two trends should be clear. First, some measures of semantic processing are less affected by the type of selection cue than others are. Concurrent interference, for example, is only found in Experiment 1 . The category effect (local targets, global ignored targets) is found in all three experiments. Negative priming is observed in some but not all experiments. This is an important result,

Table 5

Summary of Significant Effects in the Three Experiments

\begin{tabular}{lccc}
\hline & \multicolumn{2}{c}{ Location of Ignored Target } \\
\cline { 2 - 4 } & \multicolumn{3}{c}{ On Unattended } \\
\cline { 2 - 3 } Targets & On Attended & $\begin{array}{c}\text { Ignored, } \\
\text { Local }\end{array}$ & $\begin{array}{c}\text { Ignored, } \\
\text { Global }\end{array}$ \\
\hline Local & & & \\
$\quad$ Concurrent interference & $1,2,3$ & 1 & 1,3 \\
$\quad$ Negative priming & 3 & 1 & $1,2,3$ \\
$\quad$ Category effect & 2,3 & 1,3 & \\
Global & & & 1 \\
$\quad$ Concurrent interference & & & 2 \\
$\quad$ Negative priming & & & 1,3 \\
$\quad$ Category effect & &
\end{tabular}

Note-Numbers indicate which experiments yielded a significant effect in either RT or error rate. For simplicity, results in a direction opposite to those expected are treated as null results. 
for it confirms that any single measure of semantic processing may not be adequate as a basis for drawing conclusions about the extent of semantic analysis of ignored information. Manipulations that appeared to reduce semantic processing (as indicated by one measure) might have little effect on other measures. Thus, for example, global ignored targets did not lead to concurrent interference when the target was global and attention was directed either by the color of the patterns (Experiment 2) or by a spatial precue (Experiment 3). Yet the negative priming and category effects measures indicated that those ignored items were processed to the semantic level.

A second important trend is that there is no evidence that local ignored targets achieve semantic analysis unless subjects are actively attending targets defined at the local level of structure. When the target is global, concurrent interference and the category effect are not found, replicating the pattern reported by Paquet and Merikle (1988). The measure of negative priming included in the present experiments extends this result by showing that failure to show effects of these ignored targets on concurrent trials is not due to inhibition of those items. The latter was a possible explanation of Paquet and Merikle's findings.

What are the implications of these results for models of visual attention, and more specifically, for the ongoing debate between proponents of early and late selection? First, the point was raised earlier that a failure to find evidence for semantic analysis of irrelevant items is not by itself sufficient to allow a choice between early and late selection. Early selection proponents could argue that such a result supports a limited capacity attention system, while late selection proponents could counter that this merely reflects the use of a highly efficient selection cue after semantic analysis. The use of multiple indicants of nontarget processing is a strategy that can be useful in contrasting these two positions. If various measures of nontarget processing converge on the conclusion that no semantic analysis occurs, one can have more confidence in taking an early selection position than if only one measure is relied on. On the other hand, if one can show clear dissociations between the indicants used, the late selection proponents will be bolstered by the demonstration that a single measure of nontarget processing is an unreliable indicant of true semantic analysis.

Different aspects of the results here support each of these alternatives. When the ignored targets are local and attention is directed to global targets, it is clear that none of the measures show evidence for semantic analysis of local ignored targets, even when the local items are located within the object being attended. Only when attention was directed to the local level of structure was such evidence found. Such consistency of results is strong support for the claim that the local level of structure does not receive semantic analysis unless it is the focus of attention. However, when the ignored targets were global, there was compelling evidence for semantic analysis (both concurrent effects and sequential inhibition), regardless of what level of structure was being attended to. Furthermore, these measures showed dissociations compatible with the late selection view that single measures of semantic processing are insufficient.

The latter findings appear to be consistent with late selection models in this respect, but one may attempt further to distinguish between two alternative versions of late selection. The first is that ignored information simply decays when not attended to and that this prevents interference with target processing (van der Heijden, Hagenaar, \& Bloem, 1984). Alternatively, it is possible that irrelevant information must be suppressed or inhibited to prevent concurrent interference from occurring (Neill, 1977; Tipper, 1985). To address this issue, it is useful to consider the results separately for global and local ignored targets once again. When the ignored target is local, the only evidence for negative priming is obtained when attention is directed to the local level of structure (in Experiment 1). Apparently, when attention is directed to the global level, these local items need not be inhibited in order to prevent concurrent interference. When the ignored target is global, however, negative priming is often observed under conditions of local attention. And in at least one case (Experiment 2), negative priming was found in a situation in which neither concurrent interference nor a category effect occurred. Thus inhibition seems to be a potential mechanism of selection that is used in some but not all cases. More specifically, it does not seem to be required when the ignored items are local and the attended ones are global.

In summary, previous evidence (Paquet, 1988; Paquet \& Merikle, 1988) has shown that the global and local levels of structure from ignored objects are not equally capable of affecting performance. The present results replicate this finding and also show that inhibitory mechanisms of selection are not needed to explain the lack of effects of concurrent local distractors. Furthermore, making selection easier can totally eliminate evidence for semantic analysis according to one measure (concurrent interference) but leave other measures (negative priming and the category effect) unaffected. The general pattern of results is compatible with the view that an initial preattentive analysis achieves semantic processing for only the global level of structure of all patterns in a visual display.

\section{REFERENCES}

Allport, D. A., Tipper, S. P., Chmiel, N. R. J. (1985). Perceptual integration and postcategorical filtering. In M. I. Posner \& O. S. M. Marin (Eds.), Attention and performance XI (pp. 107-132). Hillsdale, NJ: Erlbaum.

BRIAND, K. A. (1989). Visual selective attention in filtering and onienting paradigms: Objects versus dimensions. Unpublished doctoral dissertation, Dalhousie University, Halifax, NS, Canada.

Briand, K. A. (1993). Efficient filtering of irrelevant global and local information when target level and location are random. Psychological Research, 55, 264-269. 
Briand, K. A., \& KLEIN, R. (1993). Selective attention to global and local structure of objects: The role of cuing dimension. Unpublished manuscript.

BroadBent, D. E. (1970). Stimulus set and response set: Two kinds of selective attention. In D. I. Mostofsky (Ed.), Attention: Contemporary theories and analysis (pp. 51-60). New York: AppletonCentury-Crofts.

Driver, J., \& Tipper, S. P. (1989). On the nonselectivity of "selective" seeing: Contrasts between interference and priming in selective attention. Joumal of Experimental Psychology: Human Perception \& Performance, 15, 304-314.

Duncan, J. (1981). Directing attention in the visual field. Perception \& Psychophysics, 30, 90-93.

ERIKSEN, B. A., \& ERIKSEN, C. W. (1974). Effects of noise letters upon the identification of a target letter in a nonsearch task. Perception \& Psychophysics, 16, 143-149.

HofFMAN, J. E. (1975). Hierarchical stages in the processing of visual information. Perception \& Psychophysics, 18, 348-354.

Kahneman, D., \& Henik, A. (1981). Perceptual organization and attention. In M. Kubovy \& J. R. Pomerantz (Eds.), Perceptual organization (pp. 181-211). Hillsdale, NJ: Erlbaum.

KINCHLA, R. A., \& WOLFE, J. M. (1979). The order of visual processing: "Top-down," "bottom-up," or "'middle-out." Perception \& Psychophysics, 25, 225-231.

Muller, H. J., \& Findlay, J. M. (1988). The effects of visual attention on peripheral discrimination thresholds in single and multiple element displays. Acta Psychologica, 69, 129-155.

MulleR, H. J., \& RABBITT, P. M. A. (1989). Reflexive and voluntary orienting of visual attention: Time course of activation and resistance to interruption. Journal of Experimental Psychology: Human Perception \& Performance, 15, 315-330.

Navon, D. (1977). Forests before trees: The precedence of global features in visual perception. Cognitive Psychology, 9, 353-383.

NeILL, W. T. (1977). Inhibition and facilitation processes in selective attention. Journal of Experimental Psychology: Human Perception \& Performance, 3, 444-450.

NeIsser, U. (1967). Cognitive psychology. Englewood Cliffs, NJ: Prentice-Hall.

PAQUet, L. (1988). L'Attention selective: L'Effet du critère de selection. L'Année Psychologique, 88, 179-195.

Paquet, L., \& Merikle, P. M. (1988). Global precedence in attended and nonattended objects. Journal of Experimental Psychology: Human Perception \& Performance, 14, 89-100.
Remington, R., \& Pierce, L. (1984). Moving attention: Evidence for time-invariant shifts of visual selective attention. Perception \& Psychophysics, 35, 393-399.

TIPPER, S. P. (1985). The negative priming effect: Inhibitory priming by ignored objects. Quarterly Journal of Experimental Psychology, 37A, 571-590.

TIPPER, S. P., \& DRIVER, J. (1988). Negative priming between pictures and words in a selective attention task: Evidence for semantic processing of ignored stimuli. Memory \& Cognition, 16, 64-70.

VAN DER HeIJDEN, A. H. C. (1981). Short-term visual information processing. London: Routledge \& Kegan Paul.

van der Heijden, A. H. C., Hagenaar, R., \& Bloem, W. (1984). Two stages in postcategorical filtering and selection. Memory \& Cognition, 12, 458-469.

\section{NOTES}

1. There were only two neutral letters in the stimulus set ( $L$ and $E$ ), and one restriction was that one of these had to appear in the relevant object and the other in the irrelevant object. On one-target trials, the second letter needed to create the compound letter pattern in the irrelevant object was either the neutral letter shown in the relevant object or the neutral letter shown in the irrelevant object.

2 . This possibility was suggested by an anonymous reviewer of an earlier version of this manuscript.

3. The fast and slow responses on concurrent trials were determined separately for the variables of target size and compatibility because we required a comparison of fast and slow concurrent trials that was not confounded with these factors. A comparison of one-target trials following fast responses to global targets with one-target trials following slow responses to local targets would obviously lead to different latencies, but this could be due to target size per se. The appropriate comparison must be between trials having targets of the same size. Similarly, suppose we compare one-target trials following fast responses on concurrent compatible trials with one-target trials following slow responses on concurrent incompatible trials. This comparison is not useful, because if performance is exceedingly slow following trials with an incompatible distractor, this might be because of the artifact under consideration, or it might be because of negative priming. For these reasons, within-subject comparisons of fast and slow responses (and subsequent performance on one-target trials) were carried out separately for the factors of target size and compatibility, and the data are collapsed across these variables in Table 4.

APPENDIX A

Global Targets: Mean Reaction Time (RT) and Error Rate (\%E) for All 15 Experimental Conditions

\begin{tabular}{|c|c|c|c|c|c|c|}
\hline \multirow[b]{3}{*}{ Trials } & \multicolumn{6}{|c|}{ Experiment } \\
\hline & \multicolumn{2}{|c|}{1} & \multicolumn{2}{|c|}{2} & \multicolumn{2}{|c|}{3} \\
\hline & RT & $\% \mathrm{E}$ & RT & $\% \mathrm{E}$ & RT & $\% \mathrm{E}$ \\
\hline \multicolumn{7}{|l|}{ Concurrent } \\
\hline Attended object, compatible & 624 & 3.9 & 563 & 4.0 & 479 & 3.0 \\
\hline Attended object, incompatible & 617 & 3.9 & 588 & 5.6 & 478 & 2.1 \\
\hline Ignored object, local, compatible & 633 & 6.3 & 576 & 3.1 & 467 & 3.1 \\
\hline Ignored object, local, incompatible & 611 & 3.9 & 567 & 2.9 & 468 & 1.6 \\
\hline Ignored object, global, compatible & 628 & 6.7 & 565 & 4.2 & 496 & 2.4 \\
\hline Ignored object, global, incompatible & 700 & 18.0 & 592 & 5.4 & 511 & 3.1 \\
\hline \multicolumn{7}{|l|}{ One Target } \\
\hline Attended object, compatible & 618 & 2.6 & 554 & 2.6 & 483 & 3.4 \\
\hline Attended object, incompatible & 612 & 1.0 & 554 & 2.1 & 450 & 1.4 \\
\hline Ignored object, local, compatible & 625 & 2.6 & 573 & 3.5 & 479 & 2.5 \\
\hline Ignored object, local, incompatible & 629 & 6.2 & 571 & 3.4 & 465 & 1.9 \\
\hline Ignored object, global, compatible & 622 & 3.6 & 579 & 5.4 & 484 & 2.6 \\
\hline Ignored object, global, incompatible & 625 & 6.8 & 583 & 3.5 & 473 & 1.2 \\
\hline Previously attended, compatible & 628 & 3.7 & 560 & 2.4 & 476 & 1.5 \\
\hline Previously attended, incompatible & 641 & 3.6 & 564 & 4.6 & 484 & 1.7 \\
\hline Baseline & 600 & 3.2 & 555 & 5.3 & 474 & 1.7 \\
\hline
\end{tabular}


APPENDIX B

Local Targets: Mean Reaction Time (RT) and Error Rate (\%E) for All 15 Experimental Conditions

\begin{tabular}{|c|c|c|c|c|c|c|}
\hline \multirow[b]{3}{*}{ Trials } & \multicolumn{6}{|c|}{ Experiment } \\
\hline & \multicolumn{2}{|c|}{1} & \multicolumn{2}{|c|}{2} & \multicolumn{2}{|c|}{3} \\
\hline & RT & $\% \mathrm{E}$ & RT & $\% \mathrm{E}$ & RT & $\% \mathrm{E}$ \\
\hline \multicolumn{7}{|l|}{ Concurrent } \\
\hline Attended object, compatible & 784 & 9.4 & 626 & 2.9 & 557 & 3.4 \\
\hline Attended object, incompatible & 823 & 14.5 & 672 & 5.5 & 641 & 15.9 \\
\hline Ignored object, local, compatible & 739 & 11.3 & 632 & 4.2 & 571 & 4.6 \\
\hline Ignored object, local, incompatible & 811 & 31.3 & 639 & 3.4 & 570 & 5.6 \\
\hline Ignored object, global, compatible & 836 & 16.8 & 681 & 5.6 & 593 & 5.9 \\
\hline Ignored object, global, incompatible & 859 & 12.5 & 652 & 4.2 & 579 & 4.9 \\
\hline \multicolumn{7}{|l|}{ One Target } \\
\hline Attended object, compatible & 760 & 9.9 & 623 & 2.6 & 578 & 4.5 \\
\hline Attended object, incompatible & 789 & 11.5 & 631 & 2.4 & 574 & 4.9 \\
\hline Ignored object, local, compatible & 817 & 6.8 & 668 & 3.4 & 596 & 4.3 \\
\hline Ignored object, local, incompatible & 800 & 13.5 & 647 & 3.9 & 558 & 3.3 \\
\hline Ignored object, global, compatible & 772 & 9.9 & 634 & 4.3 & 558 & 3.3 \\
\hline Ignored object, global, incompatible & 824 & 15.1 & 647 & 2.9 & 572 & 4.9 \\
\hline Previously Attended, compatible & 795 & 12.0 & 626 & 3.5 & 573 & 6.4 \\
\hline Previously Attended, incompatible & 808 & 13.5 & 645 & 2.2 & 573 & 5.5 \\
\hline Baseline Trials & 778 & 7.0 & 621 & 2.6 & 569 & 1.4 \\
\hline
\end{tabular}

(Manuscript received August 23, 1991;

revision accepted for publication October 15, 1993.) 